\title{
RHIC AC Dipole Design and Construction *
}

\author{
M. Bai, M. Meth, C. Pai, B. Parker \\ S. Peggs, T. Roser, R. Sanders, D. Trbojevic, A.Zaltsman \\ Brookhaven National Laboratory, Upton, NY 11973, U.S.A
}

\begin{abstract}
Two ac dipoles with vertical and horizontal magnetic field have been proposed at RHIC for applications in linear and non-linear beam dynamics and spin manipulations. A magnetic field amplitude of $380 \mathrm{Gm}$ is required to produce a coherent oscillation of 5 times the rms beam size at the top energy. We take the ac dipole frequency to be $1.0 \%$ of the revolution frequency away from the betatron frequency. To achieve the strong magnetic field with minimum power loss, an air-core magnet with two seven turn winding of low loss Litz wire resonating at $64 \mathrm{kHz}$ is designed. The system is also designed to allow one to connect the two magnet winding in series to resonate at $37 \mathrm{kHz}$ for the spin manipulation. Measurements of a half length prototype magnet are also presented.
\end{abstract}

\section{INTRODUCTION}

Two ac dipole magnets with oscillating horizontal and vertical magnetic field will be installed in RHIC in the summer of 2001. One application of the ac dipoles is to excite a coherent oscillation in the transverse planes for optics measurement and beam dynamics studies [1]. By manipulating the ac dipole in an adiabatic fashion, namely being slowly energized and de-energized, a sustained coherent oscillation with a large amplitude can be obtained and the beam emittance can be preserved after the beam manipulation [2]. This has been demonstrated in the AGS polarized proton experiments [3] where a similar device (RF dipole) was employed to induce a strong coherent oscillation in the vertical plane to avoid the beam polarization loss when crossing strong intrinsic spin resonances.

In addition, the vertical ac dipole (with horizontally oriented magnetic field) will also be used to induce an adiabatic spin flip for RHIC spin physics experiment [4]. In this application, the vertical ac dipole kicks the spin vector away from the vertical direction every time when the beam passes the magnet. When the frequency of the ac dipole oscillating magnetic field coincides with the spin precession frequency, the kicks on the spin vector from the ac dipole then add up constructively. Thus, a spin flip is induced. In RHIC polarized proton acceleration, two full Siberian snakes are installed in each ring (blue and yellow) to eliminate the spin depolarization resonance [5]. In their pres-

${ }^{*}$ The work was performed under the auspices of the US Department of Energy
Table 1: Expected ac dipole parameters for different applications

\begin{tabular}{|c|c|c|c|c|}
\hline application & $\begin{array}{c}\mathrm{B}_{m} \mathrm{~L} \\
{[\mathrm{G}-\mathrm{m}]}\end{array}$ & $\begin{array}{c}\text { center } \\
\text { frequency } \\
f_{m}[\mathrm{kHz}]\end{array}$ & $\begin{array}{l}\text { tuning } \\
\text { range } \\
{[\mathrm{kHz}]}\end{array}$ & $\begin{array}{l}\text { Duty } \\
\text { time }\end{array}$ \\
\hline spin flipper & $\geq 100$ & Vert: 37.5 & \pm 1.0 & $2.4 \mathrm{~s}$ \\
\hline $\begin{array}{c}\text { optics meas- } \\
\text {-urement }\end{array}$ & 78 & $\begin{array}{l}\text { Hori: } 63.95 \\
\text { Vert: } 63.73\end{array}$ & & $40 \mathrm{~ms}$ \\
\hline $\begin{array}{c}\text { non-linear } \\
\text { dynamics }\end{array}$ & 380 & $\begin{array}{l}\text { Hori: } 63.95 \\
\text { Vert: } 63.73\end{array}$ & \pm 1.0 & $80 \mathrm{~ms}$ \\
\hline
\end{tabular}

ence, the spin precession tune ${ }^{1}$ is independent of the beam energy.

Based on the above physics requirements, table 1 summarizes the expected ac dipole parameters.

\section{RHIC AC DIPOLE SYSTEM}

\subsection{Magnet}

To eliminate the power dissipation in the ferrite or other magnetic material, the RHIC ac dipole is designed to be an air-core magnet. each magnet consists of two coils with seven turn winding. Figure 1 is the schematic drawing of the ac dipole coil. The pseudo $\cos \theta$ shaped coil is for the concern of the field quality. It is made of a special type of wire (Litz Wire) which consists of more than 4000 individually insulated strands to minimize the ac power dissipation in the winding due to the eddy current effects. Figure 2 shows the measured ac resistance of in two half meter long ac dipole prototypes, one is made of regular stranded cable and the other is of Litz wire. The Litz wire ac resistance is almost independent on the the frequency and is significantly less than the ac resistance of the regular stranded wire.

The two ac dipole magnets are identical except the orientation of the two coils. Each magnet is 1.19 meter long. The two coils are assembled around a ceramic beam pipe of $53^{\prime \prime}$ (length) $\times 1 \frac{5}{8}^{\prime \prime}(I D) \times 1 \frac{7}{8}^{\prime \prime}(\mathrm{OD})$. The same type of beam pipe is also used in the RHIC injection kicker. The inside surface of the ceramic beam pipe is coated with a thin

\footnotetext{
${ }^{1}$ number of precession when particle finishes one circulation along the ring
} 

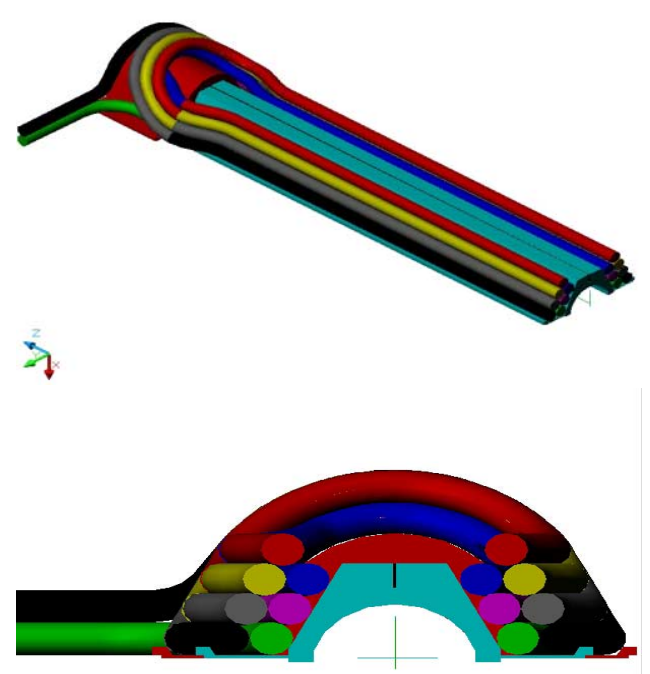

Figure 1: Schematic drawing of the ac dipole coil. The top part of the figure is the 3D side view of the coil and the lower part of the figure shows the cross section of the coil.

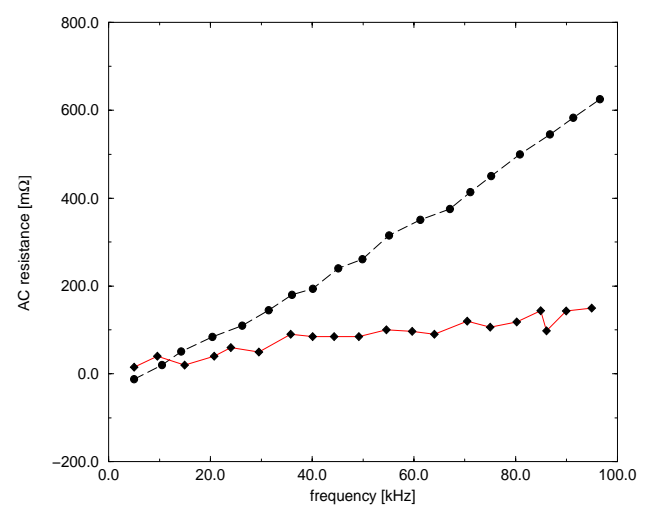

Figure 2: The dashed line is the measured ac resistance of the half meter long ac dipole made of regular stranded wire. The solid line is the measured ac resistance of the Litz wire ac dipole prototype. It is clear that the ac resistance of the Litz wire has much less dependence on the frequency than the regular stranded wire.

layer of high impedance material to reduce the collection of the static charge. Although it allows a good penetration of the oscillating magnetic field, it does not provide a good path for the image current follow. In order to minimize the beam impedance of the ac dipole, each magnet has two thin aluminum strips located at the symmetry plane of the magnetic field to provide a path for the image current flow as shown in Figure 1.

In order to switch between the two difference operation modes, namely, the two difference resonance frequencies, a contractor relay is used to allow us to switch the vertical
Table 2: ac dipole magnet parameters

\begin{tabular}{|c|c|c|}
\hline frequency & inductance & coil connection \\
\hline $37 \mathrm{kHz}$ & $104.20[\mu \mathrm{H}]$ & in serial \\
\hline $64 \mathrm{kHz}$ & $26.05[\mu \mathrm{H}]$ & in parallel \\
\hline
\end{tabular}

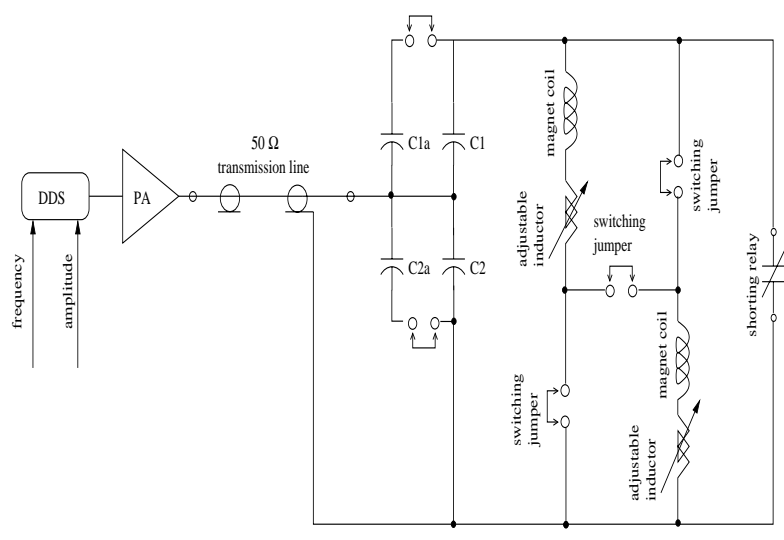

Figure 3: Schematic drawing of the ac dipole circuit diagram. $C_{1}$ and $C_{2}$ are the capacitors for the resonant frequency at $37.5 \mathrm{kHz} . C_{1 a}$ and $C_{2 a}$ are switched on for the resonance frequency at $64 \mathrm{kHz}$. The two adjustable external inductors are in series with each coil for frequency sweeping. For the RHIC 2001 run, they are not available and two shunt resistors are used instead to deliberately spoil the $\mathrm{Q}$ factor. The shorting relay is to short the circuit when the ac dipole is not in use. The linear power amplifier is driven by a DDS (Digital Direct Synthesizer) which outputs the low level amplitude and frequency modulated sinusoidal signal.

ac dipole coil connection between being in series for the spin application and being in parallel for the coherence excitation. The horizontal dipole coils are permanently connected in parallel. Table 2 lists the calculated magnet inductance.

\subsection{Electrical system}

For each RHIC ac dipole, a set of capacitors is employed to form an $\mathrm{LC}$ circuit resonated at $64 \mathrm{kHz}$ for the coherence excitation. For the vertical ac dipole, the LC circuit also contains another set of capacitors to allow the magnet to be tuned at $37.5 \mathrm{kHz}$ for the spin applications. The switch between the two different operation modes is implemented through the contact relays. Both circuits are driven through capacitive tap to load the power supply cables in their surge impedance of $50 \Omega$. Figure 3 is a schematic drawing of the ac dipole circuit diagram. In order to maintain high $\mathrm{Q}$ factor of the circuit, the capacitors are chosen to be low power loss dissipation.

To achieve $\pm 1 \mathrm{kHz}$ frequency sweeping range, an exter- 
nal inductor is employed as shown in Figure 3. Its inductance can be changed by adjusting the bias current. An amplitude feedback loop is required for each magnet to maintain a constant amplitude magnetic field as the frequency is swept.

The driving signal of the power amplifier is a low level amplitude modulated sinusoidal signal. Eq. 1 is a typical ac dipole magnetic field $B$ signal.

$$
B=B_{m}(t) \cos \left(f_{m}(t) t+\chi\right),
$$

where the amplitude profile $B_{m}(t)$ and frequency profile $f_{m}(t)$ are

$$
B_{m}(t)= \begin{cases}\frac{B_{m 0} 0}{n_{u p}} t & 0<t<n_{u p} \\ B_{m 0} & n_{u p}<t< \\ & n_{u p}+n_{f t} \\ B_{m 0}\left(1-\frac{t-n_{u p}-n_{f t}}{n_{d n}}\right) & n_{u p}+n_{f t}< \\ & t<n_{\text {total }}\end{cases}
$$

$$
f_{m}(t)= \begin{cases}f_{m 1} & 0 \geq t \leq n_{u p} \\ f_{m 1}+\frac{f_{m 2}-f_{m 1}}{n_{f t}}\left(t-n_{u p}\right) & n_{u p}<t \\ & <n_{u p}+n_{f t} \\ f_{m 2} & n_{u p}+n_{f t}< \\ & t<n_{\text {total }}\end{cases}
$$

The authors would like to thank J. Delong for providing

zero to the desired field, $n_{f t}$ is the time when the ac dipole field oscillating amplitude stays fixed, $n_{d n}$ is the time for the ac dipole to get ramped down to zero field and $n_{\text {total }}=$ $n_{u p}+n_{f t}+n_{d n}$.

The high gain power amplifier is driven by a low level sinusoidal waveform generated by a DDS (Direct Digital Synthesizer) module based on the input frequency and amplitude. The DDS module reads the inputs of the amplitude and frequency according to a event on the RHIC Time line. Since the fastest available event is at $720 \mathrm{~Hz}$, the ramping of the magnetic field oscillating amplitude has to be longer than $65 \mathrm{~ms}$ to guaranty the adiabaticity.

For the RHIC Year 2001 run, only the vertical ac dipole will be powered and commissioned as a spin flipper during the polarized proton operation. Since the external adjustable inductor will not be available for this year, a set of external resistors is used to deliberately spoil the $\mathrm{Q}$ factor to achieve $\pm 1 \mathrm{kHz}$ frequency sweeping range. A 6000 watt linear power amplifier will be used to reach 100 Gauss magnetic field. Table 3 summarizes the electrical parameters for this year.

\section{CONCLUSION}

A pair of ac dipole magnets will be installed this summer in RHIC. Both ac dipoles will be the tool to excite long lasting coherent betatron oscillations in the two transverse planes for optics measurement and beam dynamic studies. In addition, the ac dipole with horizontally oriented magnetic the DDS module, L. Hoff, J. P and B. Oerter for their help on implementing the ac dipole control system. The author would also like to thank H. Hahn for his help on the impedance issue.

Table 3: ac dipole electrical parameters for Year 2001

\begin{tabular}{|c|c|c|}
\hline & $64 \mathrm{kHz}$ & $37.5 \mathrm{kHz}$ \\
\hline capacitance $[\mu \mathrm{F}]$ & 0.245 & 0.173 \\
\hline 3 DB bandwidth $[\mathrm{Hz}]$ & 1.0 & 1.0 \\
\hline magnet voltage & 1684 volts & 1975 volts \\
\hline magnet current & $158 \mathrm{Amp}$ & $79 \mathrm{Amp}$ \\
\hline total power loss $[$ watts] & 5681.0 & 4731.7 \\
\hline coil power loss $[$ watts $]$ & 624 & 624 \\
\hline shunt resistor $\Omega$ & 300 & 500 \\
\hline Q factor & 23.6 & 16.7 \\
\hline
\end{tabular}

field will also be used as spin flipper during the polarized proton operation. The two magnets are air-core magnets with two seven turn coils. To achieve, high quality factor $\mathrm{Q}$, litz wire is used to minimize the ac power loss. For this year's RHIC run, the vertical ac dipole (with horizontally oriented magnetic field) will be commissioned as spin flipper during the polarized proton operation.

\section{ACKNOWLEDGEMENT}

\section{REFERENCES}

[1] S. Peggs, IEEE Particle Accelerator Conference and International Conference on High-Energy Accelerators, New York, 1999.

[2] M. Bai et al., Physical Review E, 5(1997).

[3] M. Bai et al., Phys. Rev. Lett. 80, 4673 (1998).

[4] T. Roser, Handbook of Accelerator Physics and Engineering, edited by A. Chao and M. Tigner.

[5] RHIC Spin Physics Design Manual 